extent macrophages, are the main inflammatory cells, with only a small number of B cells. ${ }^{6}$ Class II major histocompatibility antigens are usually not expressed in the normal resting mammary epithelium, ${ }^{7}$ although occasionally weak staining may be seen. Strong class II MHC expression in the normal breast is only seen during pregnancy and lactation. The intensity of HLA-DR staining in women with sclerosing lymphocytic lobulitis varies from weak to strong, sometimes within a single biopsy specimen. Thus the patchy epithelial expression of HLA-DR in both cases reported here is consistent with sclerosing lymphocytic lobulitis, but is not strong supportive evidence. The significance of the absence of epithelial staining by LN3 in the series of Tomaszewski $e t$ $a l$ is difficult to judge as they give limited detail of the immunohistochemical method. The association with diabetes mellitus in both of the cases reported previously and one of our cases is another similarity with the disorder in women.

The pattern and immunophenotype of the inflammation, the presence of stromal fibrosis with epithelioid fibroblasts, as well as the association with diabetes mellitus support the view that this disorder in the male breast is the equivalent of sclerosing lymphocytic lobulitis seen in women.

We thank Dr K Eustathios for supplying clinical details for patient 2 .

1 Schwartz IS, Strauchen JA. Lymphocytic mastopathy, An autoimmune disease of the breast? Am 7 Clin Pathol 1990; 93:725-30.

2 Lammie GA, Bobrow LG, Staunton MDM, Levison DA, Page G, Millis RR. Sclerosing lymphocytic lobulitis of the breast - evidence for an autoimmune pathogenesis. Histopathology 1991;19:13-20.

3 Tomaszewski JE, Brooks JSJ, Hicks D, Livolski VA. Diabetic mastopathy: a distinctive clinicopathological entity. Hum mastopathy: a distinctive
Pathol 1992;23:780-6.

4 Ashton MA, Lefkowitz M, Tavassoli FA. Epithelioid stromal cells in lymphocytic mastitis - a source of confusion with invasive carcinoma. Mod Pathol 1994;7:49-54.

5 Epenetos AA, Bobrow LG, Adams TE, Collins CM, Isaacson PG, Bodmer WF. A monoclonal antibody that detects HLA-D region antigen in routinely fixed, wax embedded sections of normal and neoplastic lymphoid tissue. $¥$ Clin Pathol 1985;38:12-17.

6 Giorno R. Mononuclear cells in malignant and benign human breast tissue. Arch Pathol Lab Med 1983;107:41517.

7 Bartek J, Petrek M, Vojtesek B, Bartkova J, Kovarik J Rejthar A. HLA-DR antigens on differentiating mammary gland epithelium and breast tumours. $B r \quad \mathcal{f}$ Cancer gland epithelium

\title{
Association between thyroid cancer of cribriform variant and familial adenomatous polyposis
}

\author{
K Hizawa, M Iida, TYao, K Aoyagi, Y Oohata, R Mibu, KYamasaki, T Hirata, \\ M Fujishima
}

Second Department of

Internal Medicine,

Faculty of Medicine,

Kyushu University,

Maidashi 3-1-1,

Fukuoka 812, Japan

K Hizawa

K Aoyagi

M Fujishima

Second Department of Pathology

TYao

First Department of

Surgery

Y Oohata

R Mibu

Division of Diagnostic

Ultrasound

KYamasaki

T Hirata

Division of

Gastroenterology,

Department of

Medicine,

Kawasaki Medical

School, Kurashiki,

Japan

M Iida

Correspondence to:

Dr Kazuoki Hizawa.

\begin{abstract}
A case of a 20 year old Japanese woman who developed thyroid cancer exhibiting unusual cribriform structures while being followed up for familial adenomatous polyposis/Gardner's syndrome is reported. The patient presented with osteomas, pigmented retinal lesions, and adenomas of the duodenum and the papilla of Vater, in addition to numerous adenomatous polyps in the colorectum. On ultrasonography, the thyroid cancer was localised to the right lobe and was identified as an irregular, internal echo tumour with a peripheral hypoechoic zone, measuring $1.8 \mathrm{~cm}$ in diameter. Histological examination of the resected tumour showed a concomitance of papillary proliferation and cribriform structures with follicles of varying sizes. These features can be distinguished from sporadic thyroid cancer.

(F Clin Pathol 1996;49:611-613)
\end{abstract}

Keywords: familial adenomatous polyposis, thyroid cancer.
Familial adenomatous polyposis (FAP)/ Gardner's syndrome is an inherited disorder caused by a germline mutation of the APC gene. It is characterised by intestinal adenomatous polyps predisposing to cancer. Recent long term, follow up studies have clarified that patients with this condition are also susceptible to malignancies involving multiple sites. ${ }^{12}$ The thyroid gland, particularly in women, is reported to be the most frequently involved organ. ${ }^{2}$ Here, we present a young Japanese woman with thyroid cancer exhibiting unusual histological features of cribriform proliferation. These features are similar to those described recently by Harach et al. ${ }^{3}$ We also review previous cases described in the literature. ${ }^{4-9}$

\section{Case report}

A 20 year old Japanese woman was admitted to hospital in July 1993 for treatment of colonic polyposis. The patient had been diagnosed as having familial adenomatous polyposis at the age of 11 years, when her mother had undergone a total colectomy for colon cancer involving adenomatosis of the colorectum. The patient's condition had been good and she had 


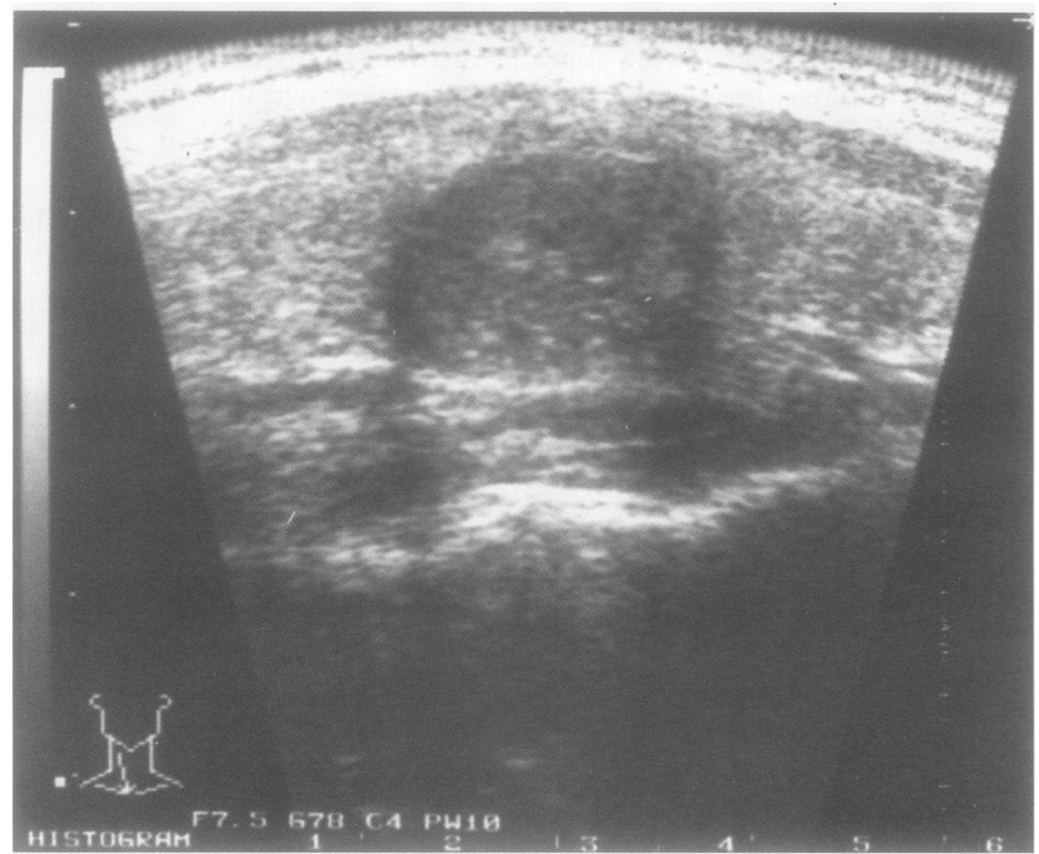

Figure 1 Ultrasonographic image showing an irregular internal echo tumour with a peripheral hypoechoic zone localised in the right lobe of the thyroid gland.

no history of exposure to radiation. Physical examination was normal, except for a nodular goitre palpable in the right thyroid gland, a small osteoma posterior to the right ear, and pigmented lesions on the right ocular fundus. There were no significant findings on laboratory tests, which included assessments of serum free thyroxin $(0.12 \mathrm{ng} / \mathrm{l})$, thyrotropin $(1.2 \mu \mathrm{U} / \mathrm{ml})$, thyroglobulin $(6.0 \mathrm{ng} / \mathrm{ml})$, and thyroxin binding globulin $(21.5 \mu \mathrm{g} / \mathrm{ml})$ concentrations. Thyroid ultrasonography using a 7.5 $\mathrm{MHz}$ concave linear array probe revealed a solitary mass with an irregular internal echo pattern, with a peripheral hypoechoic zone in the middle of the right thyroid gland (fig 1). The mass measured $1.8 \mathrm{~cm}$ in diameter. Ultrasonography of the left lobe was normal and there was no lymphadenopathy. Fine needle aspiration, guided by ultrasonography, provided material with cytology consistent with

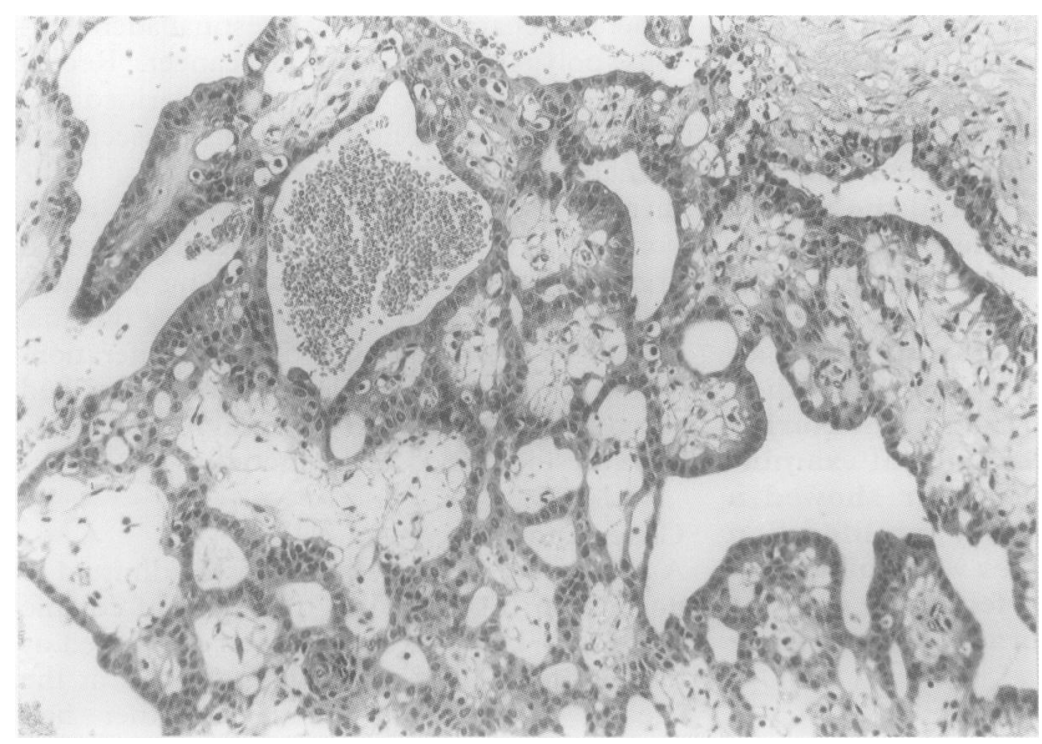

Figure 2 Histological examination of the resected specimen revealed an encapsulated thyroid cancer exhibiting cribriform structures with follicles of varying sizes (haematoxylin and eosin, $\times 190$ ). thyroid cancer. Follow up radiography of the colon revealed an increasing number of colonic adenomatous polyps, although no evidence of malignancy was found on histological examination of biopsy specimens. Gastroduodenoscopy and histology also revealed adenomatosis of the duodenum and the papilla of Vater.

In August 1993 the patient underwent right 0 hemithyroidectomy with lymph node dissec- $\frac{\mathscr{O}}{\overrightarrow{5}}$ tion following total colectomy and mucosal $\stackrel{0}{.}$ proctectomy. The resected right thyroid gland $\overrightarrow{\vec{F}}$ contained a well circumscribed, firm tumour $\frac{\vec{\sigma}}{\sigma}$ measuring $1.8 \times 1.2 \mathrm{~cm}$. Histological examination revealed an encapsulated tumour, com- $\frac{\bar{c}}{\bar{c}}$ posed of carcinoma cells with grooved nuclei $\vec{\phi}$ proliferating in a concomitant papillary and $\frac{a}{0}$ cribriform fashion with follicles of varying sizes (fig 2). There was neither lymph node $\overrightarrow{0}$ metastases nor vascular invasion. The entire $\overrightarrow{\vec{\omega}}$ mucosal surface of the resected bowel was $\stackrel{\omega}{\sigma}$ studded with numerous polyps $(>1000)$ up to $\frac{}{8}$ $1 \mathrm{~cm}$ in diameter. All of the polyps were adenomas with mild to moderate epithelial atypia, containing no cancerous foci.

Six months later, ileo-anal anastomosis with $\vec{\overrightarrow{ }}$ a J-pouch procedure was completed. The 음 patient's postoperative course has been stable, $\vec{c}$ and follow up ultrasonography of the left thyroid gland in May 1995 was unremarkable.

\section{Discussion}

The early recognition of extracolonic malignancies is of increasing importance in patients with FAP as advances in prophylactic colectomy have resulted in a reduction in the number of deaths from colorectal cancer. ${ }^{2} \stackrel{\mathbb{Q}}{\Omega}$ Iwama et al reviewed the case records of 1050 하 Japanese patients with FAP, and estimated that the relative risk of those patients developing thyroid cancer is 21 times greater than that of the general population. In addition, Plail et al 용 have indicated that the risk in women with FAP :under 35 years of age is 160 times normal. Therefore, patients with FAP should be moni- 8 tored closely for signs of thyroid cancer, particularly women.

The use of ultrasonography has improved assessment of thyroid disease, even when lesions cannot be detected on clinical examin- N ation. $^{10}$ It is the most suitable screening method because of it is simple, cost-effective $\mathrm{N}$ and highly sensitive. High resolution ultra- $\omega$ sonography can detect lesions as small as $1 \mathrm{~mm}$. In addition, fine needle aspirates $\stackrel{0}{=}$ provide direct and specific information once a $\stackrel{\oplus}{\rightarrow}$ thyroid nodule has been identified on ultrasound images. ${ }^{10}$ Our patient was accurately diagnosed with thyroid cancer on the basis of $\overrightarrow{\mathbb{Q}}$ ultrasonographic and aspiration biopsy find- $\mathbb{\nabla}$ ings.

To our knowledge, 40 cases of thyroid cancer in patients with FAP have been fully docu- $\frac{0}{0}$ mented in the English literature ${ }^{3-9}$; this estimate excludes rare cases complicated by multi- 윽 ple endocrine neoplasia. According to the literature and our own findings, thyroid cancer associated with this condition is characterised by a female preponderance $(90.0 \%)$, a papillary structure $(88.6 \%)$, and occurs in patients under 35 years of age $(83.8 \%)$. More than half 
of the tumours $(56.7 \%)$ showed multicentricity. In addition, the recognition of thyroid cancer preceded the diagnosis of intestinal polyposis in about $25 \%$ of these patients. For patients with thyroid cancer manifesting these features, examination of the colon is therefore essential.

Recently, Harach $e t a l^{\beta}$ reported four cases of thyroid cancer associated with FAP, which manifested the papillary form and also had a cribriform pattern. These authors suggest that this tumour is characteristic of patients with FAP, although it has been described as a papillary cancer in previous reports because of its structure. Our patient also manifested the cribriform features, which are distinct from thyroid cancer in the general population. This histological property may suggest a relation between thyroid cancer and a genetic abnormality associated with the APC gene. Recent genetic investigation ${ }^{11}$ suggest that mutation of the APC gene is unlikely to play a part in the development of a common sporadic form of papillary thyroid cancer. This finding may support the morphological distinction of thyroid cancer in patients with FAP.

Prognosis of patients with FAP and thyroid cancer is difficult to assess. Two patients with FAP and thyroid cancer have died. $^{7}$ In addition, thyroid cancer has recurred following surgery in two ${ }^{45}$ of 29 patients with average observation periods of 7.1 years. Both of these patients underwent lobectomy at the initial surgery. Considering the incidence of multifocal development, total or near-total thyroidectomy may be a more appropriate treatment for patients with thyroid cancer and FAP.
Nevertheless, we performed a hemithyroidectomy in the present patient to maintain postoperative thyroid function as the tumour was a solitary, encapsulated cancer which did not involve the lymph nodes. Further observation using thyroid ultrasonography is mandatory to prevent recurrence. Periodic fine needle aspiration biopsy may also be necessary.

The authors thank Dr Atsuhiko Sakamoto (Associate Professor, University of Tokyo) for his valuable comments on the histological evaluation of this patient.

1 Iwama T, Mishima Y, Utsunomiya J. The impact of familial adenomatous polyposis on the tumorigenesis and mortality at the several organs. Ann Surg 1993;21 7:101-8.

2 Giardiello FM, Offerhaus GJA, Lee DH, Krush AI Tersmette AC, Booker SV, et al. Increased risk of thyroid and pancreatic carcinoma in familial adenomatous polyposis. Gut 1993;34:1394-6.

3 Harach HR, Williams GT, Williams ED. Familial adenomatous polyposis associated thyroid carcinoma: a distinct tous polyposis associated thyroid carcinoma: a distinct
type of follicular cell neoplasm. Histopathology 1994; type of follic

4 Smith WG. Familial multiple polyposis: research tool for investigating the etiology of carcinoma of the colon. Dis Colon Rectum 1968;11:17-31.

5 Lee FI, MacKinnon MD. Papillary thyroid carcinoma associated with polyposis coli: a case of Gardner's syndrome. Am $\mathcal{f}$ Gastroenterol 1981;76:138-40.

6 Plail RO, Bussey HJR, Glazer R, Thomson JP. Adenomatous polyposis: an association with carcinoma of the tous polyposis: an association with

7 Piffer S. Gardner's syndrome and thyroid cancer: a case report and review of the literature. Acta Oncol 1988; 27:413-15.

8 Kelly MD, Hugh TB, Field AS, Fitzsimons R. Carcinoma of the thyroid gland and Gardner's syndrome. Aust NZ $\mathcal{F}$ Surg 1993;63:505-9.

9 Bell B, Mazzaferri EL. Familial adenomatous polyposis (Gardner's syndrome) and thyroid carcinoma. Dig Dis $S c i$ 1993;38:185-90.

10 Mazzaferri EL, Santos ET, Rofagha-Keyhani S. Solitary thyroid nodule: diagnosis and management. Med Clin North Am 1988;72:1177-211.

11 Curtis L, Wyllie AH, Shaw JJ, Williams GT, Radulescu A, DeMicco C, et al. Evidence against involvement of APC mutation in papillary thyroid carcinoma. Eur $\mathcal{f}$ Cancer 1994;30A:984-7. 